ARTICLE

\title{
CRISPR-Cas9 globin editing can induce megabase- scale copy-neutral losses of heterozygosity in hematopoietic cells
}

\author{
J. Boutin (10) 1,2,3,4,9, J. Rosier 1,2,4,9 , D. Cappellen (1) 1,2,4,6, F. Prat ${ }^{1,2,4}$, J. Toutain ${ }^{5}$, P. Pennamen (1) ${ }^{5}$, J. Bouron ${ }^{5}$, \\ C. Rooryck1,5, J. P. Merlio (1) 1,6,7, I. Lamrissi-Garcia1,2,4, G. Cullot1,2,4, S. Amintas 1,2,4,6, V. Guyonnet-Duperat ${ }^{8}$, \\ C. Ged ${ }^{1,2,3,4}$, J. M. Blouin 1,2,3,4 E. Richard1,2,3,4, S. Dabernat ${ }^{1,2,3,4}$, F. Moreau-Gaudry (1) ${ }^{1,2,3,4,8,9 凶}$ \& \\ A. Bedel $1,2,3,4,9$
}

CRISPR-Cas9 is a promising technology for gene therapy. However, the ON-target genotoxicity of CRISPR-Cas9 nuclease due to DNA double-strand breaks has received little attention and is probably underestimated. Here we report that genome editing targeting globin genes induces megabase-scale losses of heterozygosity ( $\mathrm{LOH}$ ) from the globin CRISPR-Cas9 cut-site to the telomere $(5.2 \mathrm{Mb})$. In established lines, CRISPR-Cas9 nuclease induces frequent terminal chromosome 11p truncations and rare copy-neutral $\mathrm{LOH}$. In primary hematopoietic progenitor/stem cells, we detect $1.1 \%$ of clones $(7 / 648)$ with acquired megabase LOH induced by CRISPR-Cas9. In-depth analysis by SNP-array reveals the presence of copy-neutral LOH. This leads to 11p15.5 partial uniparental disomy, comprising two Chr11p15.5 imprinting centers (H19/IGF2:IG-DMR/IC1 and KCNQ1OT1:TSS-DMR/IC2) and impacting $\mathrm{H} 19$ and IGF2 expression. While this genotoxicity is a safety concern for CRISPR clinical trials, it is also an opportunity to model copy-neutral-LOH for genetic diseases and cancers.

\footnotetext{
${ }^{1}$ Bordeaux University, Bordeaux, France. ${ }^{2}$ INSERM U1035, Biotherapy of Genetic Diseases, Inflammatory disorders and Cancers, Bordeaux, France. ${ }^{3}$ University Hospital Bordeaux, Biochemistry Laboratory, Bordeaux, France. ${ }^{4}$ Laboratory of Excellence, Gr-Ex, Bordeaux, France. ${ }^{5}$ Bordeaux University, MRGM INSERM U1211, CHU de Bordeaux, Service de Génétique Médicale, Bordeaux, France. ${ }^{6}$ University Hospital Bordeaux, Tumor Biology and Tumor Bank Laboratory, Bordeaux, France. ${ }^{7}$ INSERM U1053, Bordeaux Research in Translational Oncology, Bordeaux, France. ${ }^{8}$ INSERM US 005-CNRS UMS 342-TBM-Core, Bordeaux University, Bordeaux, France. ${ }^{9}$ These authors contributed equally : J. Boutin, J. Rosier, F. Moreau-Gaudry, A. Bedel. ${ }^{凶}$ email: francois.moreau-gaudry@u-bordeaux.fr
} 
asin and colleagues ${ }^{1}$ provided evidences of engineered nucleases interest for the induction of double-strand break (DSB) in order to be applied to the treatment of human diseases. CRISPR-Cas9 has become a universal and powerful method for the precise genome editing of many organisms ${ }^{2,3}$ to model diseases, study genotype-phenotype relationships and improve gene therapy. The modification of human hematopoietic stem/progenitor cells (HSPCs) by CRISPR-Cas9 has rapidly led to clinical trials (clinical trial.gov: NCT03655678/NCT03745287). However, nucleasemediated DSBs might present drawbacks as previously described with I-Sce1, ZFN and TALEN ${ }^{4}$. Unwanted OFF-target effects have been widely described. While DNA-DSBs can lead to undesirable small insertions/deletions (Indels) and even chromosomal rearrangements (translocations, inversions and deletions) $)^{5-7}$, guideRNA (gRNA) design optimization and the use of highly specific Cas9 nucleases can control them. However, safety issues regarding genome ON-target integrity lack in-depth exploration. In addition to small Indels, a single ON-target DSB can lead to large deletions up to several kilobases in size, symmetrical or not, in mouse embryos $^{8,9}$, in mouse hematopoietic progenitors, in human immortalized differentiated cells ${ }^{10}$ and human embryos ${ }^{11}$. Recently, we reported that CRISPR-Cas9 can even cause megabase-scale chromosomal truncations targeting Chromosome 10q (Chr10q) in two human cell lines and in human primary fibroblasts deficient for the tumor suppressor $\mathrm{p} 53^{12}$. These large mono-allelic genomic deletions were confirmed in human colorectal carcinoma HCT116 cells targeting Chr18q arm terminal deletion ${ }^{13}$, in human primary iPSC targeting Chr7 and Chr2 $1^{14}$, and very recently in early human embryos with risk of aneuploidy ${ }^{14,15}$. In addition, more complex rearrangements were present ${ }^{8,10-12}$, suggesting that the diversity of potential deletion outcomes is vast. These genomic alterations have not been reported until now because standard genotyping methods frequently miss them. Reassuringly, to date these genomic rearrangements have not been linked to deleterious consequences for patients.

It is currently unknown whether large alterations can occur after DSB in human HSPCs, the relevant clinical target cells for gene therapy protocols. Indeed, HSPCs benefit from superior p53dependent capacity to prevent the accumulation of genetic lesions, repair them, and avoid their propagation to daughter cells ${ }^{16-18}$. In this work we observe that CRISPR-Cas9 nuclease induces frequent terminal chromosome $11 \mathrm{p}$ truncations in cell line and rare extra-large terminal copy-neutral loss-of-heterozygosity in HSPCs resulting in partial uniparental disomy of the 11p15.4-11p15.5 imprinted region altering $H 19$ and IGF2 gene expression.

\section{Results}

Globin gene targeting induces extra-large Chr11p LOH by chromosomal terminal deletions in HEK cells. As we already reported truncations on Chr10q by targeting $U R O S^{12}$, we first evaluated whether such an event also occurs on Chr1lp when targeting the globin genes in HEK293T cells. These cells are known to have low p53 activity, a favorable condition not only for editing ${ }^{17,18}$ but also for genomic instability. We designed three gRNA sets (Fig. 1a). The first one (protocol \#1) targets the sixth codon of wild type $H B B$, which is mutated in sickle-cell disease (green lightning). The two other sets (protocols $\# 2^{19}$ (blue lightning) and $\# 3^{20}$ (black lightning)) are related to those previously published in successful preclinical studies for $\beta$-hemoglobinopathies gene therapy. CRISPR-Cas9 targeting by protocols \#2 and \#3 increases hemoglobin $\mathrm{F}(\mathrm{HbF})$ synthesis, recapitulating the hereditary persistence of fetal hemoglobin (HPFH)-associated mutations. Protocol \#2 targets a 13-nt sequence present in the promoters of the $H B G 1$ and $H B G 2$ genes $^{19}$. Protocol $\# 3$ uses 2 gRNAs to disrupt a $13.6 \mathrm{~kb}$ genomic region encompassing the $\delta$ - and $\beta$-globin genes and a putative $\gamma-\delta$ intergenic $\mathrm{HbF}$ silencer ${ }^{20}$ (Fig. 1a). We transfected each gRNA set with the ribonucleoprotein (RNP) Cas9 nuclease. After transfection, ICE (Inference of CRISPR Edits) analysis of the Sanger sequences confirmed the presence of a high rate of indels with all gRNAs $(83 \%, 63 \%$, and $60 \%$ for \#1, \#2 and \#3, respectively). To evaluate Chr1lp arm genomic integrity, we first performed fluorescent in situ hybridization (FISH) on HEK293T cells 4 days after editing (mainly trisomic for Chr11). We used a Chr11-specific orange sub-centromeric probe $(\mathrm{O})$ and a Chrl1p-specific sub-telomeric green probe $(\mathrm{G})$ at $5.2 \mathrm{Mb}$ from the globin cluster. Blind deep FISH analysis by an automated system revealed that the cells displaying a $3 \mathrm{O} / 2 \mathrm{G}$ profile increased from $7.3 \%$ (before transfection) to $17.2 \%, 11.9 \%, 20.8 \%$ after protocols $\# 1$, \#2 and \#3, respectively (Fig. 1b day 4 and Supplementary Figs. $1-3,>550$ cells per condition). At the same time, Chr11 2O/3G cell percentages and FISH analysis of another chromosome (Chr2) to monitor technical noise were low and stable (around 1\% in all conditions) (Fig. 1b and Supplementary Fig. 4). Around 5\% of cells were disomic for Chr11 in control cells and the prevalence was stable in edited cells, which is not in favor of induced loss of the entire chromosome. Finally, targeting another chromosome (Chr10) with nuclease did not impact 3O/2G Chr11-cell percentages, indicating that Chr11-specific sub-telomeric probe hybridization loss increases at day 4 were specific genetic outcomes due to Cas9 nuclease use in Chr11p (Fig. 1c, left). Regardless of the gRNAs and the protocols, higher frequencies of unbalance in Chr11p copies suggested that these events were likely independent of the location or the number of DSBs. Sub-telomeric green probe losses were less frequent in protocols \#1 and \#3 at D18, but still persisted even after numerous cell divisions (at around $10 \%$ in all conditions without significant difference between protocols, $p=0.838$ two-sided Chisquare tests) and was significantly higher than in unedited cells (NT, non-transfected: 6.2\%) (Fig. 1b right, day 18). In contrast, Chr2 DNA FISH at day 18 showed stability of this chromosome (Supplementary Fig. 4). These sub-telomeric probe losses could be consistent with extra-large terminal deletions of Chr11 (5.2 Mb) downstream of the globin locus, induced by nuclease-mediated DSB.

To confirm this hypothesis and estimate the incidence of these outcomes, we carried out cellular subcloning and picked up 203 clones $(54,64,54,31$ from control and protocols \#1-2-3, respectively, Fig. 2a). To detect megabase deletions, we screened for homozygosity two single-nucleotide polymorphisms (SNP, rs2735691/rs10835611) in $R R M 1$, heterozygous before editing and located $1.1 \mathrm{Mb}$ telomeric from the Cas9 breakpoint. While only $1.9 \%(1 / 54)$ of cells lost RRM1 SNP heterozygosity in the control cells, $20.3 \%(13 / 64), 18.5 \%(10 / 54)$ and $25.8 \%(8 / 31)$ of clones exhibited $\mathrm{LOH}$ in protocols \#1 to \#3, respectively, without significant difference between the protocols (Fig. 2a). 25/27 of the clones with RRM1 SNP LOH (92.6\%) screened by FISH lost one sub-telomeric green probe $(3 \mathrm{O} / 2 \mathrm{G})$ in favor of a Chr11p terminal deletion (Fig. 2b and Supplementary Table 1). Array-CGH (Array Comparative Genomic Hybridization) identified the location of the globin genes targeted by CRISPR-Cas9 as the start of the breakpoint delimiting this 5.2-megabase terminal deletion in HEK293T cells and confirmed these terminal deletions, whatever the protocol used (Fig. 2b). Interestingly, 2/27 clones (7.4\% of RRM1 LOH clones) kept 3O/3G signals, suggesting a copy-neutral LOH by mitotic recombination confirmed by CGHarray (Fig. 2b and Supplementary Table 1).

To conclude, in HEK293T cells, CRISPR-Cas9 nuclease induced frequent terminal Chr11p truncations and rare copyneutral $\mathrm{LOH}$.

None of the top-10-predicted off-target sites were situated on Chr11 (Supplementary Table 2), suggesting that these genetic outcomes were not due to additional off-target cut in the Chr11p. 
a.

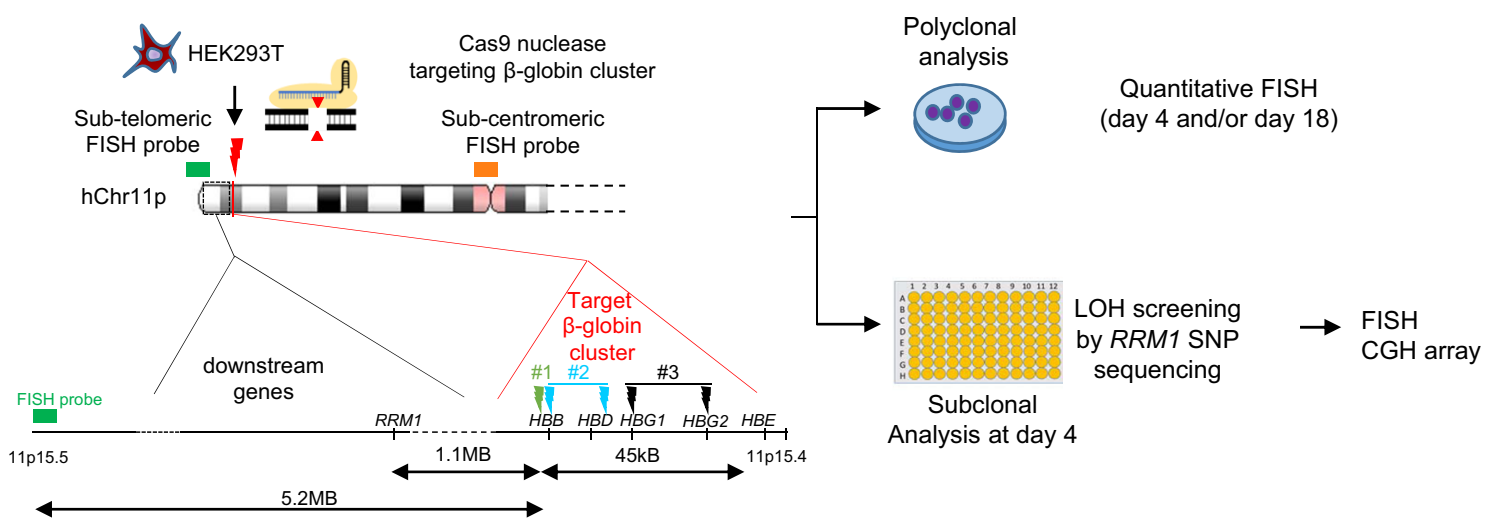

b.
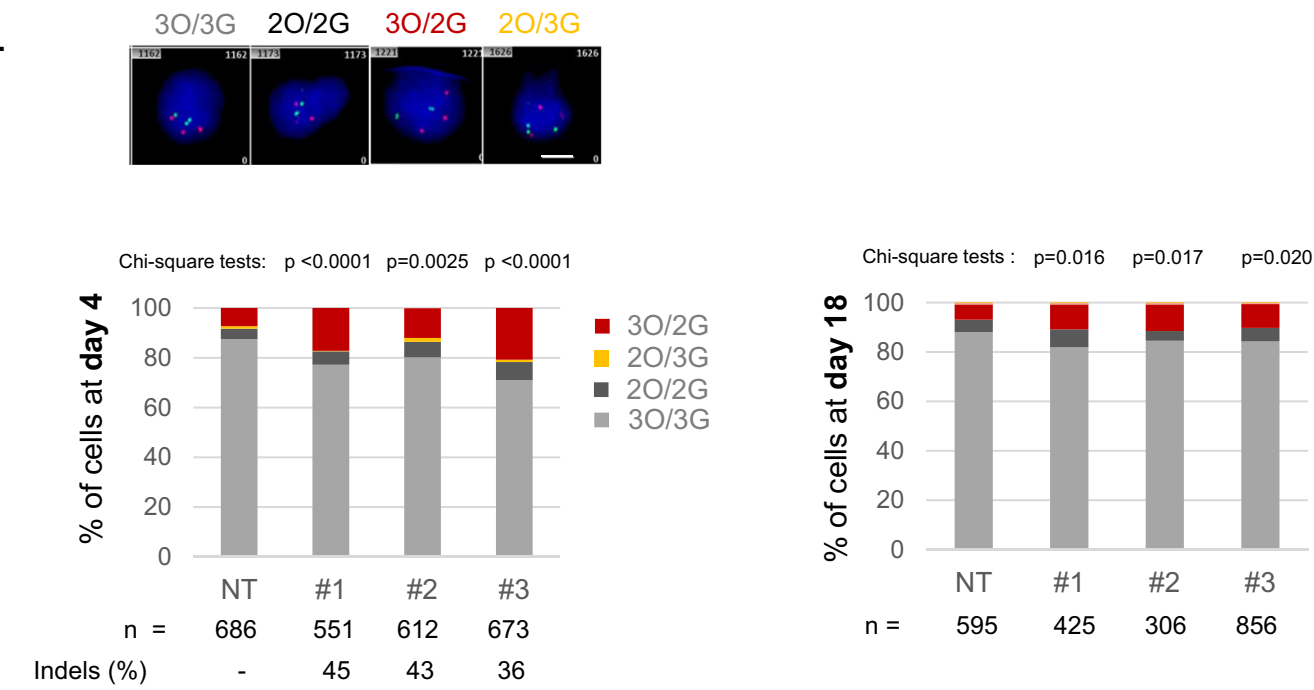

c.

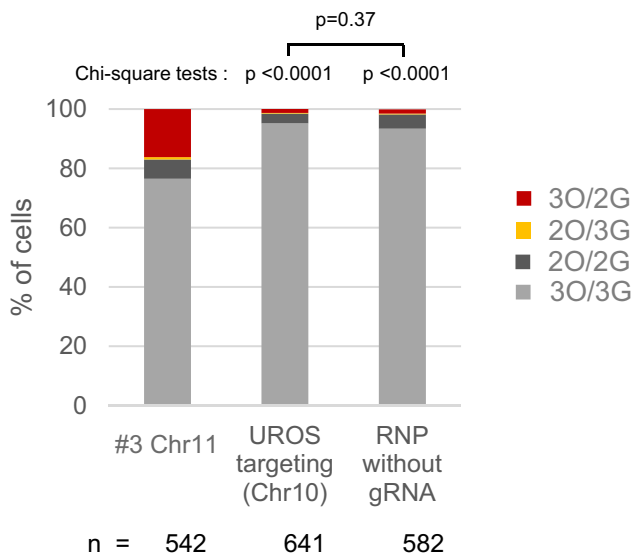

To clarify this prediction, we used HiFi Cas9, which is known to drastically reduce off-target cuts. HiFi Cas9 also increased the frequency of loss of the sub-telomeric probe, confirming that these chromosomal rearrangements were not caused by off-target effects (Fig. 1c right). Frequency of $3 \mathrm{G} / 2 \mathrm{O}$ cells with $\mathrm{HiFi}$ was twofold lower compared to protocol \#3 using regular Cas 9 but with a cut-off efficiency also reduced by half (in our experiment, $31 \%$ of indels with $\mathrm{HiFi}$ versus $61 \%$ with wild type Cas9). Taken together these data demonstrate that induced $\mathrm{LOH}$ are not dependent to off-target activity of Cas9 nuclease.

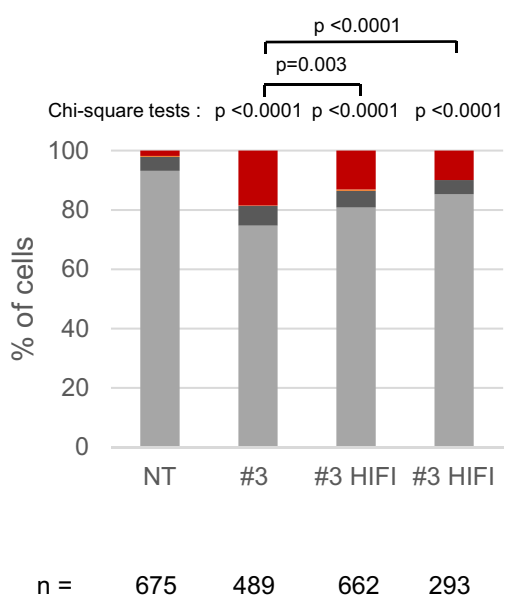

CRISPR-Cas9-mediated globin gene targeting can induce extra-large Chr11p CN-LOH in human hematopoietic stem/ progenitor cells (HSPCs). We then investigated whether these putative side-effects occurred in the clinically relevant HSPCs using $\mathrm{CD}_{3} 4^{+}$cord-blood cells. We repeated protocols \#1 and \#3 in diploid HSPCs by inducing one or two cuts, respectively (Fig. 3a). FISH analyses at D18 revealed slight increases in $20 / 1 \mathrm{G}$ cells with both protocols in both experiments $(+1.3 \%$ and $+3.55 \%$ for \#1 and \#3, respectively, versus non-transfected (NT) cells, without a significant difference between the two protocols 
Fig. 1 CRISPR-Cas9-mediated globin gene targeting induces Chr11p terminal deletions in HEK cells. a Experimental design. HEK293T cells edited with Cas9 nuclease RNP and sets of gRNA corresponding to protocols \#1 to \#3. Map of $\beta$-globin genes, telomeric downstream SNP genes (RRM1) and FISH probes. $\mathrm{LOH}$, loss of heterozygosity; SNP, single-nucleotide polymorphism; FISH, fluorescent in situ hybridization; CGH, comparative genomic

hybridization. b Illustrative Chr11 DNA-FISH in HEK cells. Scale-bar: $5 \mu$ m. Polyclonal Chr11 DNA-FISH analysis at D4 (left) and at D18 (right) after editing with protocols \#1 to \#3. NT: non-transfected. O, Orange sub-centromeric probe; G sub-telomeric Green probe. 30/3G (in gray), 30/2G (in red), 2O/3G (in yellow), and 20/2G (in black) signal percentages are quantified. 30/2G frequencies after protocols 1-3 were compared to non-transduced cells on same day by two-sided Chi-square tests. $n$ correspond to analyzed cells from each polyclonal pool (1 per protocol). c left: Polyclonal Chr11 DNA-FISH analysis at D4 after editing with protocol \#3 (Chr11), without gRNA or targeting another chromosome (UROS locus in Chr10). 3O/2G frequencies targeting Chr10 or without guide RNA were compared to protocol \#3 on same day. 3O/2G frequencies targeting Chr10 or without guide RNA were compared by two-sided Chi-square tests. Right: Polyclonal Chr11 DNA-FISH analysis at D4 after editing with protocol \#3 (Chr11), and with protocol \#3 using HiFi Cas9. 3O/3G, 3O/3G, 2O/3G and 2O/2G signal percentages were quantified. 30/2G frequencies with protocol \#3 using HiFi Cas9 or Cas9 were compared to non-transduced cells by two-sided Chi-square tests. 30/2G frequencies with protocol \#3 using HiFi Cas9 were compared to protocol \#3 using Cas9 by two-sided Chi-square tests. O: orange sub-centromeric Chr11 probe, G: green sub-telomeric Chr11p probe. $\mathrm{n}$ correspond to analyzed cells from each polyclonal pool (1 per protocol). Source data are provided as a Source Data file.

Fig. 3b, Supplementary Table 3, Supplementary Figs. 5 and 6). Chr11 1O/2G and Chr2 2G/1O cell percentages (Fig. 3b, c) did not increase. On the contrary, green probe losses $(2 \mathrm{O} / 1 \mathrm{G})$ were suggestive of $11 \mathrm{p}$ terminal deletions, but the high background noise of $2 \mathrm{O} / 1 \mathrm{G}$ in non-transduced cells makes the analysis complicated and international cytogenetic guidelines consider that the increase must be greater than $5 \%$ to be considered significant ${ }^{21}$. To improve sensitivity and conclude, we subcloned HSPCs by FACS cell sorting at day 4 post-editing. We first screened $\mathrm{LOH}$ in $\mathrm{CD} 34^{+}$clones by analyzing 4 SNPs in RRM1 and CARS1 loci, 1.1 and $2.2 \mathrm{Mb}$ telomeric to the globin cluster. We did not find any SNP loss in subclones after protocol \#1 but we were able to analyze only 79 clones and guide RNA activity was low (indel rate: 10\%). As LOHs seem to be DSB frequencydependent, we chose protocol \#3 for extensive cloning experiments. We performed two independent experiments of protocol \#3 (indel rates of 86 and $88 \%$ ), revealing the presence of two clones that lost one allele of the four SNPs $(1 / 104,1 / 107$, in \#3.1, $\# 3.2$, respectively, Fig. 3d, f). To be sure that these results were not due to the use of valproic acid (VPA, which improves CD34 ${ }^{+}$cell subcloning) and AMAXA nucleofector enhancer (enh, recommended by the manufacturer to increase editing efficiency), the same experiments were performed without VPA (VPA-/enh+) or the enh (VPA+/enh-). Clonal analysis revealed the presence of one clone with RRM1 SNPs losses (at $1.1 \mathrm{Mb}$ from the cut-site) and KCNQ1 SNP loss (at $2.5 \mathrm{Mb}$ from the cut-site), in the VPA - enh + condition (1/41 clones). The frequency of LOH was similar to that of the control VPA+/enh $+(1 / 50$ clones) (Fig. $4 \mathrm{a}$, $b, c)$. We did not find any LOH clones in VPA+/enh- condition (0/47 clones). Therefore, we removed both VPA and enh and increased the number of analyzed clones to establish whether the presence of $\mathrm{LOH}$ was dependent on enh. We obtained 3/299 colony-forming cells (CFC) with $\mathrm{LOH}$ including RRM1 and KCNQ1 loci (Fig. 4a, c), confirming the absence of VPA and enh bias. Taken together, in primary hematopoietic progenitor/stem cells, we detected $1.1 \%$ of clones (7/648) with acquired megabase LOH induced by CRISPR-Cas9.

For two clones (34.8 and 34.15) we have the chance to have enough material to explore in-depth these $\mathrm{LOH}$ induced by CRISPR-Cas9. Unexpectedly, array-CGH did not show any loss of genomic material (Fig. 3e), suggesting that these megabase-scale $\mathrm{LOH}$ were not due to terminal chromosomal deletions but to the occurrence of a copy-neutral loss of heterozygosity (CN-LOH), also called partial uniparental disomy. CGH/SNP-array analysis validated this hypothesis and mapped a single specific induced $\mathrm{CN}$ $\mathrm{LOH}$ (iCN-LOH) in both clones spanning $5.2 \mathrm{Mb}$ from the globin gene cut to the telomere (Fig. $5 \mathrm{a}$ and Supplementary Fig 7). Sanger sequencing of SNPs along the Chr11p arm again confirmed these unexpected extra-large $\mathrm{CN}-\mathrm{LOH}$ in primary hematopoietic cells probably due to mitotic recombination (Supplementary Fig. 8). To more precisely map the start of $\mathrm{LOH}$, we carried out Sanger sequencing of the breakpoint of the two clones. Clone 34.15 analysis revealed a homozygous 4-base pairs deletion, suggesting that the $\mathrm{CN}-\mathrm{LOH}$ starts exactly at the CRISPR-Cas9-cut-site. Two different edited alleles were present in clone 34.8, suggesting that $\mathrm{CN}$-LOH starts between the cut-site and the first probe of the SNP array at $0.4 \mathrm{Mb}$ (Supplementary Fig. 9a). We chose additional SNPs nearer to the CRISPR cut-site and sequenced them. We found an additional SNP (not studied by SNP array) located $0.18 \mathrm{Mb}$ from the cut-site on the telomeric side. It became homozygous in the two CD34 clones, demonstrating that the $\mathrm{LOH}$ is already present. As expected, the SNP on the centromeric side $(0.06 \mathrm{Mb}$ from the globin cut-site) were still heterozygous (supplementary Fig. 9b). Taken together, these data highlight the presence of $\mathrm{CN}-\mathrm{LOH}$ induced by CRISPR-Cas9 in HSPC starting in the surroundings of the DSB.

Functional consequences of iCN-LOH. As the 11p15.4-11p15.5 locus is subjected to parental genomic imprinting 22 , we analyzed the methylation status of H19 (a non-coding RNA tumor suppressor with maternal expression), IGF2-AS (insulin-like growth factor II gene, pro-oncogenic gene expressed only from the paternal allele) and the KCNQ1OT1 imprinting centers in three $\mathrm{LOH}$-positive clones compared to five $\mathrm{LOH}$-negative clones. Normal cells harbor both unmethylated and methylated alleles of the H19, IGF2-AS and KCNQ1OT1 regions from paternal and maternal alleles (Fig. 5b, c and Supplementary Fig. 10). Some HSPC clones (34.8 and 34.15) with CN-LOH exhibited bi-allelic methylation of the H19 Differentially Methylated Region (DMR)/ proximal promoter and the IGF2-AS DMR imprinting centers, and a bi-allelically unmethylated KCN1QOT1 locus, indicative of partial paternal uniparental disomy impacting these imprinting centers. One other HSPC LOH-clone (34.40) showed an opposite imprinting profile indicative a partial maternal uniparental disomy showing that both profiles are possible without parental bias in vitro (Fig. 5b, c and Supplementary Fig. 10). Taken together, based on the haplotype, we obtained seven clones including three paternal duplications $(34.8,34.15$, and 34.37$)$ and four maternal duplications $(34.38 ; 34.40 ; 34.175$ and 34.190$)$. We then evaluated the transcriptional modifications due to abnormal methylations in two LOH clones with partial paternal uniparental disomy (34.8 and 34.15) and four negatives clones. H19 expression analysis of both 34.15 and 34.8 clones revealed silencing of the $H 19$ gene, consistent with the paternal uniparental disomy (UPD) of this locus and complete methylation of the gene in these cells. On the contrary, methylation of IGF2-AS resulted in the variable but significant slight induction of IGF2 compared to negative clones (Fig. 5d). 

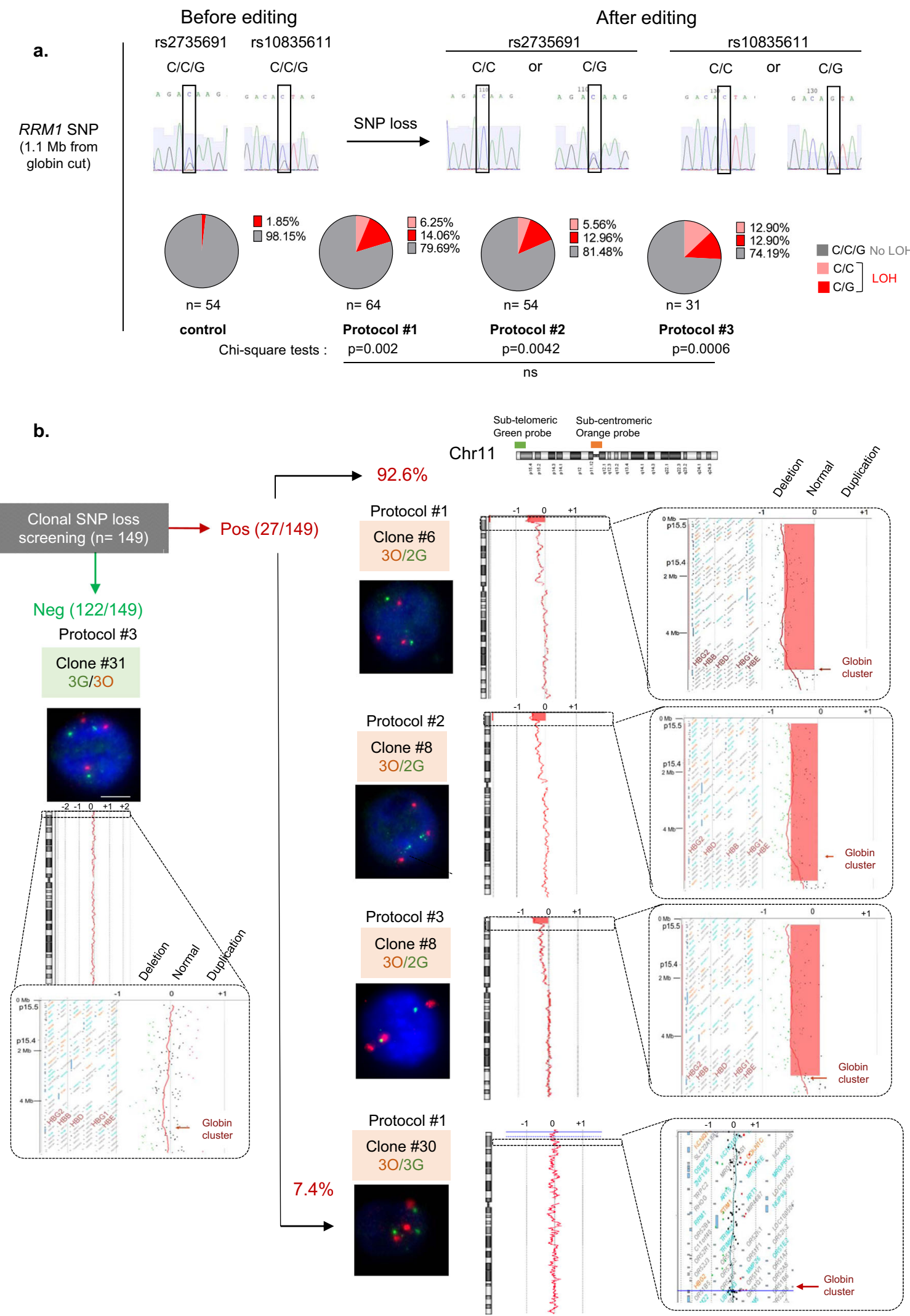

Fig. 2 Confirmation and mapping of Chr11p terminal deletions. a RRM1/SNP sequencing after subclonal analysis of HEK293T at D4. C/C/G genotype in RRM1 (in gray) on two SNP positions for NT cells. After editing, clones presented SNP-LOH with C/C (in pink) or C/G genotype (in red). Frequencies of SNP losses are indicated in pie chart for each protocol. LOH frequencies with protocols 1-3 were compared to non-transduced cells by two-sided Chi-square tests. Indels frequency was $63,8356 \%$ for protocols 1-3, respectively, $n$ correspond to analyzed cells from each polyclonal pool (1 per protocol). b Chromosome analysis in HEK293T clones depending on SNP screening. Left, DNA-FISH (Scale-bar: $5 \mu \mathrm{m}$ ) and array-CGH of one LOH-negative clone. Right, DNA-FISH and array-CGH of LOH-positive clones from protocols \#1-3. Deleted area is in red. $n$ correspond to analyzed SNP loss cells (from protocols \#1-3). Source data are provided as a Source Data file. 


\section{a.}
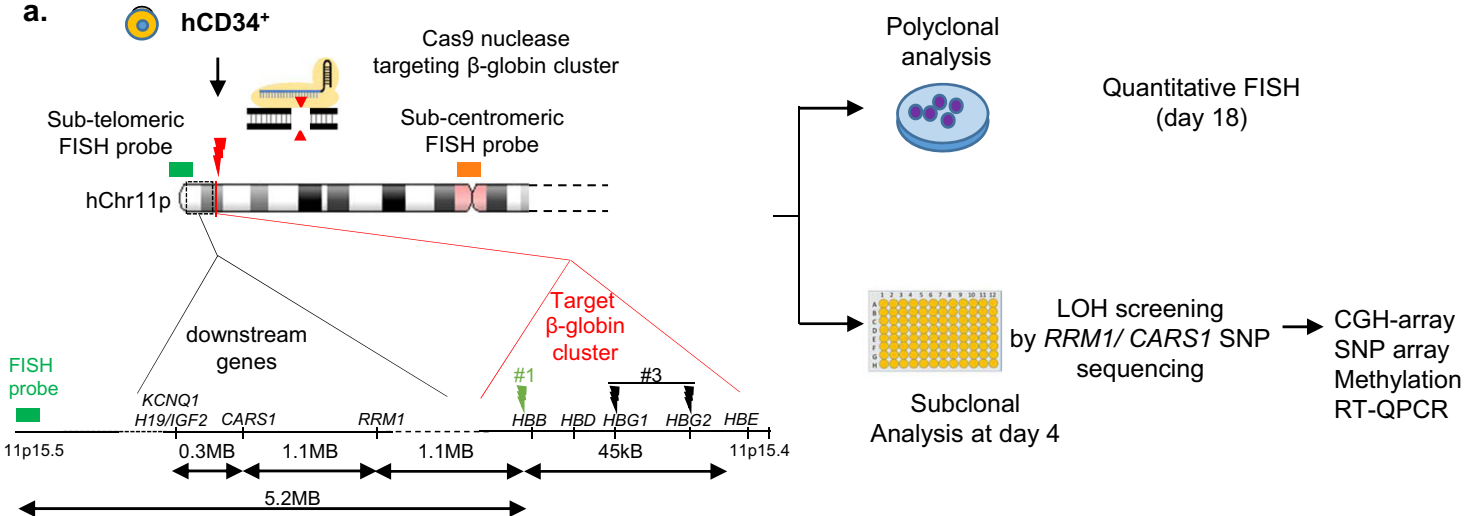

b.
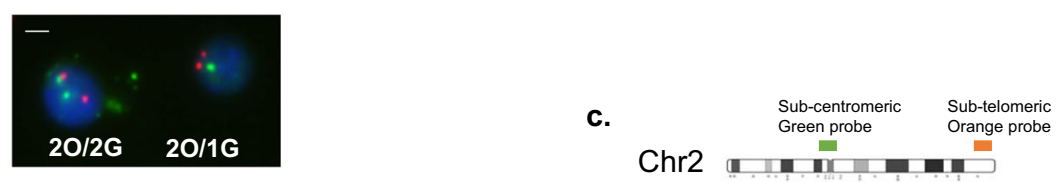

D18-Chr11 FISH

D18-Chr11 FISH

D18-Chr2 FISH

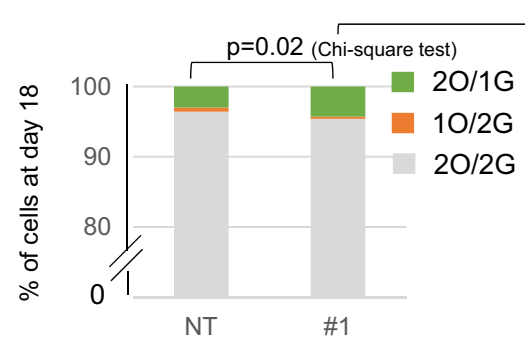

ns
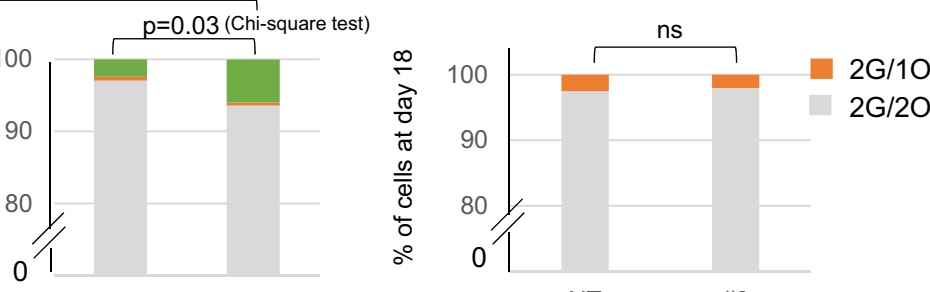

Indels (\%) - 17

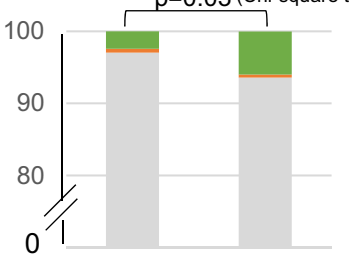

NT \#3

NT

\#3

$-\quad>90$

100

100

$\mathrm{n}=1353 \quad 1608$

d.
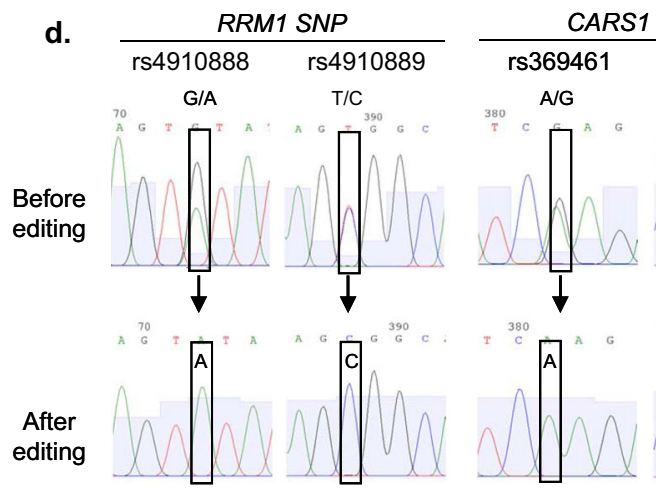

973

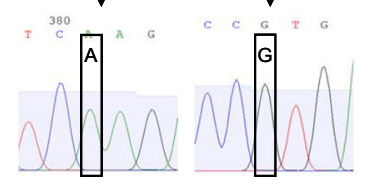

rs399565

A/G
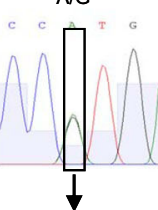

e.

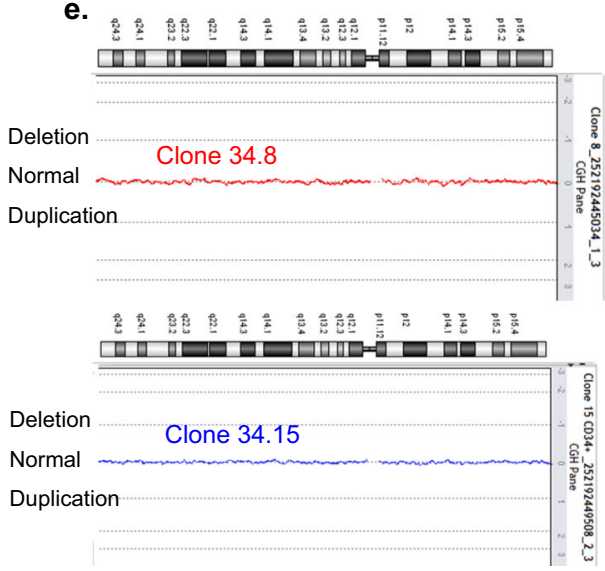

f.

LOH-positive clone frequencies

\begin{tabular}{llcc}
\cline { 2 - 4 } Protocol & $\# 1$ & $\# 3.1$ & $\# 3.2$ \\
Indels (\%) & 10 & 86 & 88 \\
& $0 / 79$ & $1 / 104$ & $1 / 107$ \\
& & Clone 34.8 & Clone 34.15
\end{tabular}

\section{Discussion}

In this study, we focused on the extra-large ON-target events that may occur when using CRISPR-Cas9 nuclease in the quest to establish appropriate quality-control measures when editing primary and animal cells. We report an ON-target genotoxicity of
CRISPR-Cas9 nuclease in HSPCs. In these clinically relevant cells, we discovered that DNA DSB can lead in vitro to megabase-scale iCN-LOH. We subcloned HSPSCs from four independent transfections with protocol \#3. We found $1.1 \%$ of clones $(7 / 648)$ with acquired megabase LOH induced by CRISPR-Cas9, 
Fig. 3 CRISPR-Cas9-mediated globin gene targeting can induce Chr11p copy-neutral-LOH (iCN-LOH) in HSPC. a Experimental design human CD34+ from cord blood edited with Cas9 RNP and sets of gRNA corresponding to protocols \#1 and \#3. Map of $\beta$-globin genes, telomeric downstream SNP genes (RRM1, CARS1 and KCNQ1), telomeric imprinted genes (H19 and IGF2) and FISH probes. b Top panel illustrative Chr11 DNA-FISH in hCD34+ cells. Scale-bar: $5 \mu \mathrm{m}$. Bottom panel, polyclonal Chr11 DNA-FISH analysis at D18 of edited cells with protocols \#1 (left) and \#3 (right). Median of each Chr11 profile (2O/2G (in gray), 2O/1G (in green), 10/2G (in orange)) in non-transfected cells as control (NT, Non-Transfected) and edited cells with protocols \#1 and \#3 ( $n=$ 7). O: orange sub-centromeric Chr11 probe, G: green sub-telomeric Chr11p probe. 2O/1G frequencies were compared between conditions by two-sided Chisquare tests. $\mathrm{n}$ correspond to analyzed $\mathrm{hCD} 34^{+}$cells for each protocol (pooled from three distinct transfections). c Polyclonal Chr2 DNA-FISH analysis at day 18 of edited cells (in Chr11) with protocol \#3 or non-transfected cells. O: orange sub-telomeric Chr2 probe, G: green sub-centromeric Chr2 probe. 2G/ 10 (in orange) frequencies were compared between conditions by two-sided Chi-square tests. $n$ correspond to analyzed hCD34+ cells for each protocol. d RRM1/CARS1 SNPs sequencing after subclonal analysis of CD34+ cells at D4, illustrating SNP-LOH observed in clones 34.8 and 34.15 from protocols \#3.1 and \#3.2. e Chromosome analysis of two HSPC clones with LOH by array-CGH (34.8 and 34.15). f LOH-positive clone frequencies for protocols \#1, \#3.1 and \#3.2. Source data are provided as a Source Data file.

suggesting that these events are not so rare, considering that millions of edited cells needed to be transplanted. While no loss of genetic material occurs, these events should not be underestimated. Indeed, they may reveal recessive mutations. Cancer cells frequently acquire a growth advantage through CN-LOH by duplicating activating mutations in oncogenes or loosing wildtype alleles of tumor suppressor genes in many cancers ${ }^{23,24}$ including leukemia ${ }^{25}$.

Recent literature suggests that this process could be locus and cell type-independent. Davis et al. ${ }^{26}$ recently described CN-LOH due to CRISPR-Cas9-mediated DSB in cancer cell lines targeting Chr11 but at another locus 11p13 (CD44). Importantly, these events were also observed in human-induced pluripotent stem cells (iPSC) targeting Chr7 and Chr $21^{14}$ and are still debated in human embryos ${ }^{15,27}$. Obviously, the consequences will likely be locus-dependent.

Here, we observed a $5.2 \mathrm{Mb}$ iCN-LOH comprising 167 genes, notably the imprinted $H 19$ and CDKN1C (cyclin dependent kinase inhibitor 1C) tumor suppressors and the IGF2 oncogene. We found that HSPCs with iCN-LOH harbor a bi-allelic paternal methylation pattern encompassing the imprinted 11p15.4-11p15.5 tumor suppressor domain, which contains the maternally expressed CDKN1C and $\mathrm{H} 19$ and the paternally expressed prooncogenic IGF2. CN-LOH-associated disomy of the methylated or unmethylated alleles led to either complete inactivation or enhanced expression of the implicated genes. Among the seven $\mathrm{LOH}$ clones, three showed paternal isodisomy. The presence of a paternal profile could be deleterious, with $H 19$ silencing and IGF2 slight induction. Single-cell RNA-seq analysis in thousands of clones will be informative to confirm transcriptional deregulation of H19 and IGF2. Indeed, paternal isodisomy is a mechanism that is known to predispose to tumors in Beckwith-Wiedemann syndrome ${ }^{28}$. Spontaneous LOH occurs unfrequently in human cells $(<0.01 \%)^{26}$. The high frequency of in vitro LOH starting at the cut-site described in this work cannot be the result of chance. Supplementary in-vivo experiments (graft in immunodeficient mice) will be required to evaluate (i) the graft capability of these rearranged cells, (ii) the long-term phenotypic consequences, and (iii) the putative selective advantage of paternal clones.

Targeting BCL11A for the induction of fetal hemoglobin ${ }^{29}$ is an alternative therapeutic approach for hemoglobinopathies. Its genotoxicity will need to be evaluated and compared to globin targeting. Interestingly, there is no imprinting center downstream of the BCL11A cleavage site and it could be safer. More generally, in light of our results, it is important to study the cancer predisposition genes distal to the DSB, notably the tumor suppressor genes whose heterozygous inactivating mutations would initiate or promote oncogenesis upon LOH targeting the functional allele.

We also demonstrate here that globin gene editing can induce frequent extra-large chromosomal deletions and rare $\mathrm{CN}-\mathrm{LOH}$ in HEK cells. Our result are in accordance with previous publications reporting the risk of chromosomal truncation in cancer cell lines ${ }^{12,13}$. Altogether, these studies highlight a nonlocus-dependent risk. Such large-scale rearrangements with CRISPR-Cas9 were recently described in human iPSC $^{14}$ and human embryos ${ }^{15}$. We did not succeed in isolating truncated HSPC clones. We cannot state formally whether these Chr11p15.4-pter deletions occurred or not. If CRISPR-induced chromosomal deletions in HSPCs do exist, this genotoxic risk is under $0.4 \%$. Primary cells could not tolerate haploidy over an extra-large region. As also suggested by three recent papers ${ }^{17,18,30}$, we previously demonstrated that the risk of chromosomal terminal deletion depends on p53 invalidation human primary fibroblastic cells ${ }^{12}$. HSPCs could benefit from p53 activity to prevent this risk ${ }^{16}$.

Further studies will be required to evaluate whether downregulation or mutations of TP53 in HSPCs induce chromosomal terminal deletion risk. It is possible that $\mathrm{iCN}-\mathrm{LOH}$ is a survival repair response to the loss of an allele in p53-proficient cells. The mechanism of $\mathrm{CN}-\mathrm{LOH}$ post CRISPR-Cas9-mediated DSB is probably consistent with Break-induced replication (BIR) recently described in eukaryotic cells ${ }^{31}$. It involves extensive DNA synthesis from DSB to the telomere ${ }^{32}$ contrary to classical gene conversion with only a short kilobase-scale patch of DNA synthesis in the neighboring of the $\mathrm{DSB}^{33}$. DNA synthesis is imprecise during BIR process. This unusual DNA repair pathway can be responsible of frequent genetic instability ${ }^{31}$. A study of the mechanisms that modulate $\mathrm{LOH}$ induced by CRISPR-Cas9 would likely elucidate this issue.

In conclusion, our findings reveal an unexpected and unexplored form of genotoxicity that could be critical for the safety of CRISPR-Cas9 gene therapies. These induced CN-LOHs (iCNLOHs) are easily missed by standard quality controls such as locus sequencing, FISH and even array-CGH. In parallel, chromotrypsis has been reported as an ON-target consequence of CRISPR-Cas9 genome editing ${ }^{34}$. The community now needs to develop technologies without subcloning to detect all ON-target rearrangements like $\mathrm{CN}-\mathrm{LOHs}$ or megabase deletions for clinical purposes. Future work should be aimed not only at detecting these iCN-LOHs but also at understanding their biological roots and reasons for occurrence as well as biological roots and phenotypic consequences. DSB-free genome editing with single nickase or base editors or nuclease-based gene therapy in iPSC with safe corrected clone sorting might be answers ${ }^{35-37}$.

\footnotetext{
Methods

Cell culture. Human embryonic kidney (HEK) cell line HEK293T (ATCC, Manassas, VA, USA) was maintained in Dulbecco's modified Eagle's medium (DMEM), low glucose (1G.L-1), L-glutamine (1G.L-1) and pyruvate (Gibco by Lifetechnologies ${ }^{\mathrm{TM}}$, Carlsbad, CA, USA) supplemented with $10 \%$ fetal bovine serum, $100 \mathrm{U} / \mathrm{mL}$ penicillin, and $100 \mu \mathrm{g} / \mathrm{mL}$ streptomycin (all from EurobioTM, Courtaboeuf, France). Human CD34+ HSPCs were isolated from the cord blood of healthy donors from Bagatelle Hospital, Bordeaux, France, according to the ethical
} 
a.

\begin{tabular}{|c|c|c|c|c|}
\hline & $\mathrm{LOH}-1$ & itive $c$ & frequ & ies \\
\hline Protocol & \#3.3 & \#3.3 & \#3.3 & \#3.4 \\
\hline Indels & $73 \%$ & $69 \%$ & $88 \%$ & $56 \%$ \\
\hline VPA/Enh & $+/+$ & $+/-$ & $-/+$ & $-/-$ \\
\hline $\begin{array}{l}\mathrm{LOH} \\
\text { Frequency }\end{array}$ & $\begin{array}{c}1 / 50 \\
\perp\end{array}$ & $0 / 47$ & $\stackrel{1 / 41}{\downarrow}$ & $\stackrel{3 / 299}{1}$ \\
\hline Clone & 34.38 & & 34.37 & $\begin{array}{l}34.40 \\
34.17 \\
34.19\end{array}$ \\
\hline
\end{tabular}

b.

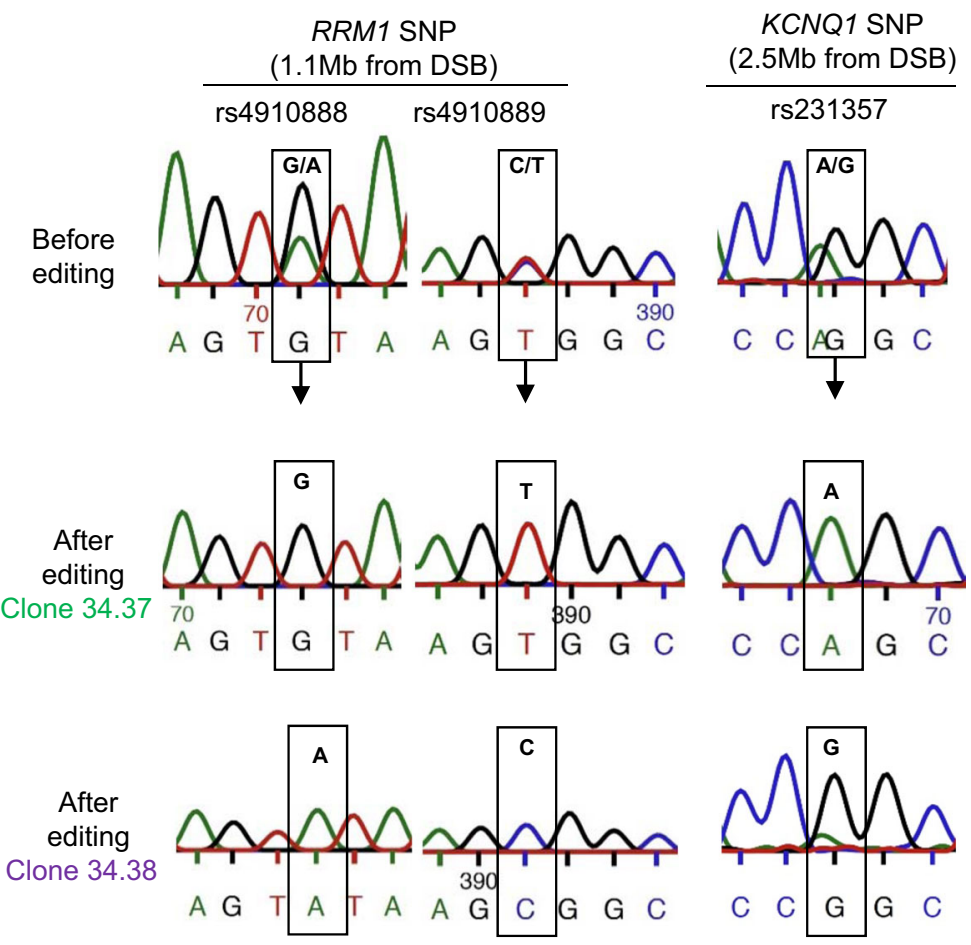

c.

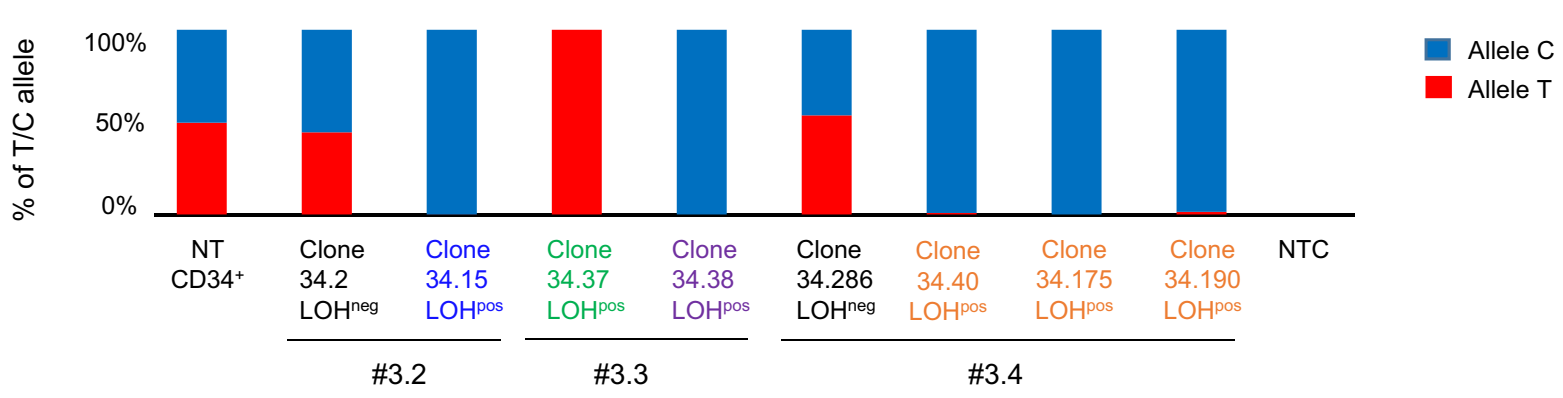

NTC

NT CD $34^{+}$

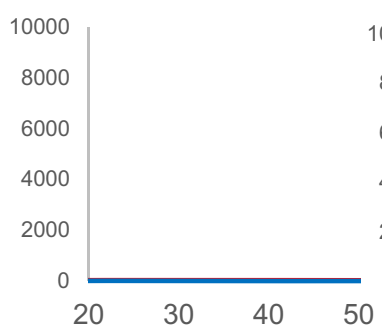

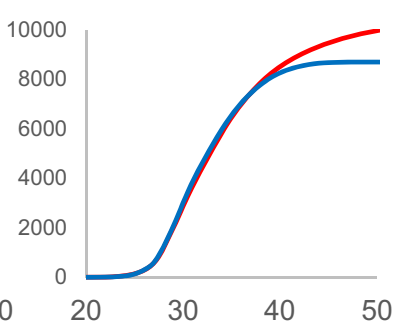

Clone 34.37

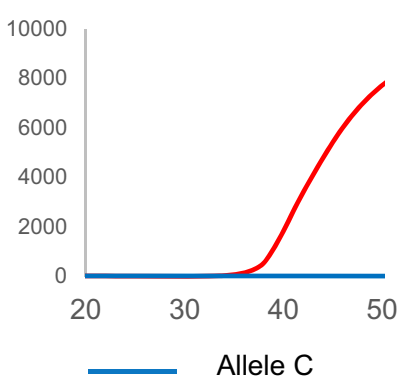

Clone 34.38

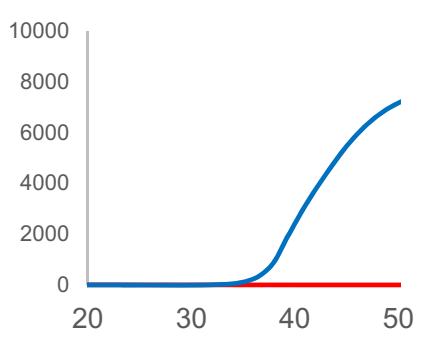

Allele T

Fig. 4 CD34+ LOH clone presence is not dependent of VPA and enhancer. a LOH-positive clone frequencies in experiments with or without valproic acid (VPA) and transfection enhancer (enh). b Illustrative Sanger sequencing of 2 RRM1 SNPs at $1.1 \mathrm{Mb}$ from the cut-site (rs4910888 and rs4910889) and KCNQ1 SNP at 2.5 Mb from the cut-site (rs231357). c Allele-specific qPCR of RRM1 (rs4910889) to determine presence or absence of SNP with illustrative curves (low panel). Non-transduced CD34+ (NT), clone 34.2 and 34.286 without LOH are heterozygous with T/C alleles (allele C in blue and allele T in red). Presence of single allele revealed LOH in numerous clones. NTC, No template control. Source data are provided as a Source Data file.

Institutional review board of Bagatelle Hospital, (Maison de Santé Protestante de Bordeaux Talence FRANCE) and with the mother's informed consent. Briefly, mononuclear cells were isolated by Ficoll gradient. CD34+ cells were purified according to the manufacturer's instructions (Human CD34-Positive Selection kit II ref 17865 from Stem Cell Technologies) and purity was analyzed by flow cytometry using phycoerythrin-conjugated anti-CD34 antibody (clone 561 Biolegend (San Diego CA, USA) $25 \mu \mathrm{g} / \mathrm{mL}$ lot \# B2044487 $5 \mu \mathrm{L} /$ test). Cryopreserved $\mathrm{CD}^{+}{ }^{+}$cells were thawed and cultured in expansion medium consisting in Stem Span SFEM (Stem Cell Technologies) supplemented with Flt3-L (50 ng/mL), SCF $(50 \mathrm{ng} / \mathrm{mL})$, human TPO $(50 \mathrm{ng} / \mathrm{mL})$, human IL3 $(20 \mathrm{ng} / \mathrm{mL})$ and human IL6 (10 
a.

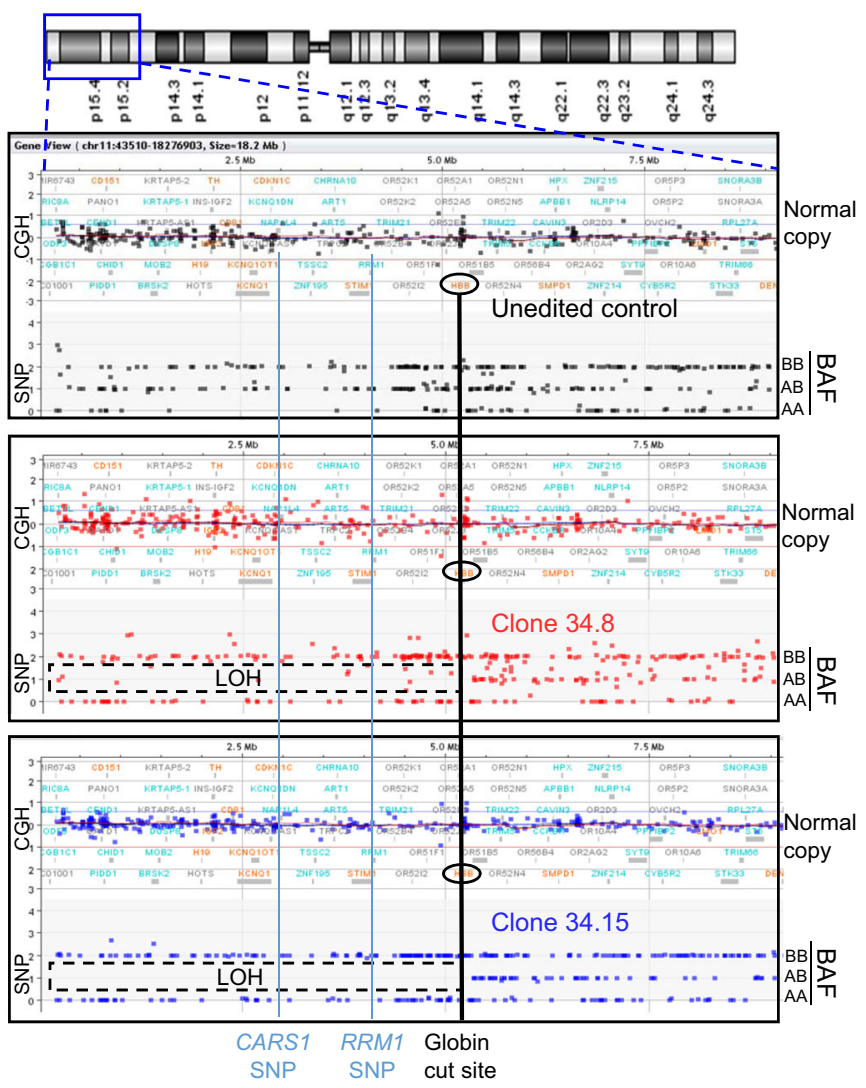

b. DNA methylation status

$\square$ Unmethylated alleles

Methylated alleles

H19 (DMR)

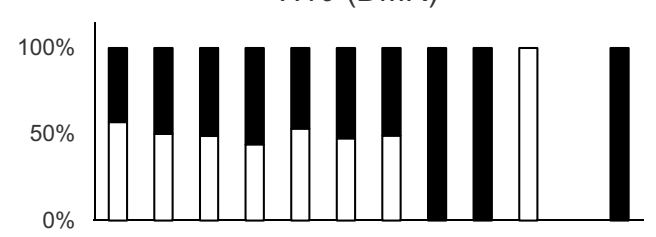

H19 proximal promoter
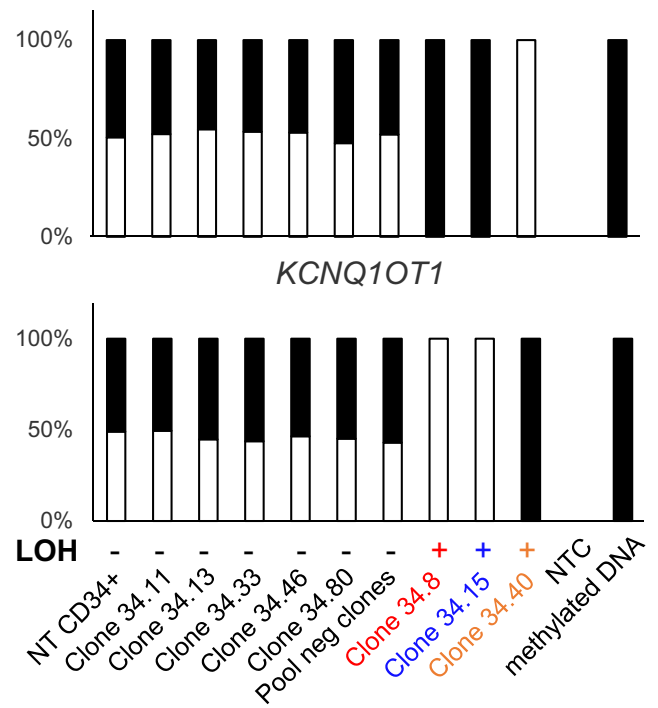

c.

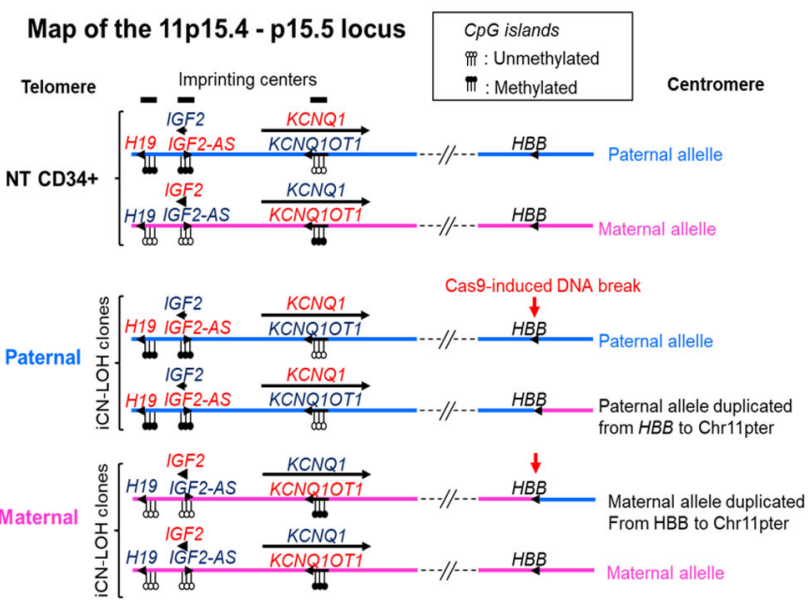

d.
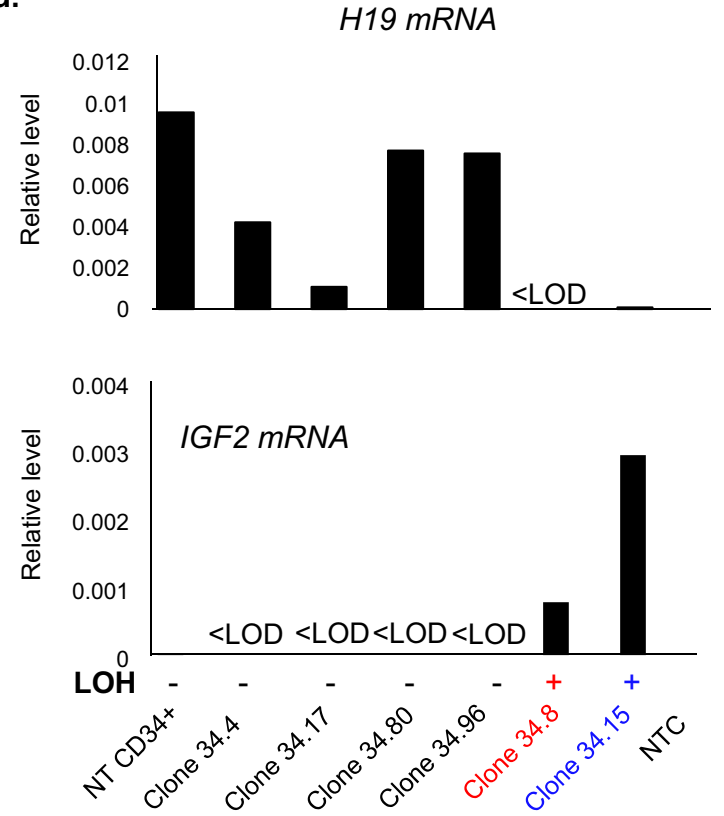

ng/mL) (all from Peprotech), StemRegenin 1 (SR1) $(1 \mu \mathrm{M})$ (Stem Cell Technologies), VPA valproic acid $(500 \mu \mathrm{M})$ (Sigma-Aldrich) (that stimulates proliferation and pluripotency of HSPC) ${ }^{38}$ and $100 \mathrm{U} / \mathrm{mL}$ penicillin, and $100 \mu \mathrm{g} / \mathrm{mL}$ streptomycin (Eurobio). Both cells were cultured at $37^{\circ} \mathrm{C}$ and with $5 \% \mathrm{CO}_{2}$ in a humidified chamber. Two days after thawing $\mathrm{CD}_{3} 4^{+}$cells were transfected with Cas 9 RNP (see below). For cellular clonal analysis, transfected cells were isolated and sorted by BD FACS Aria 4 days after transfection. They were cultured onto 96-well plates (Corning@), Tewksbury, MA, USA) and cultured as described above (cloning efficiency 50\%). SNP screening analysis was performed 3 weeks after subcloning SNP-analyses and methylation required at least 2 months expansion (clones 34.8 and 34.15). For the \#3.4 experiment, CD $34^{+}$cells were plated in $35-\mathrm{mm}$ tissue culture dishes at 150 and 450 cells $/ \mathrm{mL}$ with $1 \mathrm{~mL}$ of methylcellulose medium (Stemcell Technologies, MethoCult $\mathrm{H} 4034$ Optimum) and cultured at $37^{\circ} \mathrm{C}$ in a humidified atmosphere containing $5 \% \mathrm{CO}_{2}$. After 10 days, individual colonies were subsequently picked from plates and washed in PBS to remove all the methylcellulose. Cells were digested with proteinase $\mathrm{K}$ in lysis buffer $(10 \mathrm{mmol} / \mathrm{L}$ Tris-Cl, $\mathrm{pH}$ 


\section{Fig. 5 CRISPR-Cas9-Induced Chr11p copy-neutral-LOH (iCN-LOH) impacts methylation of imprinting centers and imprinted genes expression in}

HSPCs. a CGH/SNP-array: Log 2 Ratio and BAF (B-allele frequency) in control-HSPC cells and two edited LOH-positive-clones, zoomed on Chr11p15. Globin cut-site location is indicated by a black line. RRM1 and CARS SNP losses are indicated by a blue line. LOH are framed. $\mathbf{b}$ Percentages of unmethylated and methylated alleles of H19 differentially methylated region (H19 DMR), H19 gene proximal promoter and KCNQ1OT1 (KCNQ1 opposite strand/antisense transcript 1) in NT-CD34+ (non-transduced parental CD34+ cells), in five edited clones without LOH (34.11, 34.13, 34.33, 34.36, 34.80), in a pool of negative LOH edited clones, in 3 clones with LOH (34.8, 34.15 and 34.40) analyzed by quantitative real-time methylation-specific PCR (qMSP). in vitro methylated DNA and No DNA (NTC) were used as positive and negative controls. ND: non determined because of lack of DNA cell material. $\mathbf{c}$ Diagram of Chr11p15.4-p15.5 band with position of imprinted genes and CRISPR-Cas9-induced DNA-DSB (red arrow) in HSPCs. Paternal and maternal alleles in light blue and pink, respectively. Unmethylated and methylated CpG islands represented by white and black circles. Genes expressed from parental allele in dark blue, gene silenced on parental allele in red. Normal paternal and maternal allele from NT-CD34+ cells and alleles from paternal and maternal iCN-LOH clones are represented. $\mathbf{d} H 19$ and IGF2 mRNA expression in NT-CD34+ cells, in four edited LOH-negative clones $(34.4,34.17,34.80$, and 34.96) and two LOH-positive clones (34.8 and 34.15). Results are normalized using TATA-box binding protein (TBP) housekeeping gene. LOD, limit of detection. Quantification is represented by mean and SEM from the same sample measured repeatedly. H19 mRNA is not or hardly detectable in LOH-positive clones. IGF2 mRNA is only detectable in LOH-positive clones. Source data are provided as a Source Data file.

8.0, $50 \mathrm{mmol} / \mathrm{L} \mathrm{KCl}, 2.5 \mathrm{mmol} / \mathrm{L} \mathrm{MgCl} 2,0.5 \%$ Tween $20,100 \mathrm{mg} / \mathrm{mL}$ proteinase $\mathrm{K}$ ) at $56^{\circ} \mathrm{C}$ for $2 \mathrm{~h}$, followed by a 10 -min exposure at $95^{\circ} \mathrm{C}$.

Editing tools. HEK-293T and CD34 ${ }^{+}$cells were transfected by electroporation using the 4D-Nucleofector system AMAXA (Lonza ${ }^{\circledR}$, Bale, Switzerland) with SF Cell Line $4 \mathrm{D}$-Nucleofector ${ }^{\circledR}$ in association with the DG-150 program and the DO100 program, respectively, according to the manufacturer's instructions. To form RNP complex, Cas9 protein was complexed to crRNA:tracrRNA according to the manufacturer's instructions. Then, complexes were incubated for $20 \mathrm{~min}$ at room temperature before electroporation. Cas 9 protein (Alt-R ${ }^{\circledR}$ S.p. Cas9 Nuclease V3 or HiFi Cas9 V3 nuclease) and crRNA (Alt-R ${ }^{\circledR}$ CRISPR-Cas9 crRNA) were purchased from Integrated DNA Technologies (IDT, Coralville, IA, USA). Briefly, $10^{6}$ HEK293T cells or 150,000 primary CD34+ cells were nucleofected with $17 \mu \mathrm{g}$ of nuclease-Cas $9 / \mathrm{cr} /$ Tracr RNA complex (RNP) and $5 \mu \mathrm{M}$ of Alt-R ${ }^{\circledR}$ Cas 9 Electroporation Enhancer. Cells were then seeded onto 6-well plates (Corning ${ }^{\odot}$, Tewksbury, MA, USA) and cultured as described above.

SNP analysis by Sanger sequencing and allele-specific quantitative PCR. We used dbSNP (NCBI, nih.gov) to screen frequent SNPs in telomeric location from the beta-globin locus. We selected and tested SNPs in RRM1, CARS1 and KCNQ1 to suspect or confirm Chr11p LOH. We recovered five SNPs from SNP-array data in telomeric location from the beta-globin locus to confirm Chr11p LOH SNParray results (Supplementary Fig. 8) and 2 framing the globin cut-site (Supplementary Fig. 9). Genomic DNA was extracted for each clone by using Nucleospin ${ }^{\circledR}$ Tissue (Macherey-Nagel, Duren, Germany) according to the manufacturer's protocol. The genomic regions flanking SNPs were amplified by PCR (HotStarTaq Plus DNA polymerase, Qiagen ${ }^{\circledR}$, Venlo, Netherlands) with adequate primers (Supplementary Table 5). Design of primer was made using Primer-BLAST web tool (Primer designing tool (nih.gov).). The primer annealing temperature was calculated with a Tm calculator from New England Biolabs NEB (Tm Calculator v1.13.0; https://tmcalculator.neb.com/\#!/main). PCR products were purified with Nucleospin ${ }^{\circledR}$ Gel and PCR Clean-up (Macherey-Nagel) and sequenced. Alternatively, SNP genotyping was also performed by real-time quantitative PCR analysis (CFX Connect device, Biorad $^{\circledR}$ ) of genomic DNA with a common reverse primer and SNP allele-specific forward primers (the $3^{\prime}$ end nucleotide in bold is allele-specific, the underlined nucleotide in italic being a deliberate $\mathrm{C} / \mathrm{T}$ mismatch introduced to enhance specificity). SNP rs4910889 primers, RRM1 gene (locus 11p15.4): Forward primer allele T: CCTGAGTGCCACAGTCCCAGT; Forward primer allele C: CTGAGTGCCACAGTCCCAGC; Common revererse primer: AGGCAATTCCACAGTATGGGT. SNP $r \bar{s} \overline{2} 31357$ primers, KCNQ1/KCNQ1OT1 gene (locus 11p15.5-p15.4): Forward primer allele A: ACGTTTCATAGTCAGAC AAACCCA; Forward primer allele G: CGTTTCATAGTCAGACAAACCCG; Common reverse primer: GGGGAGGATGAAGTTAGCTGA. To validate these real-time PCR assays, PCR was performed in parallel with flanking primers encompassing the SNPs of interest, and PCR products were processed by Sanger sequencing. Quantitative allele-specific PCR analyses were quantified using the comparative MNE (Mean Normalized Expression) method, ${ }^{39}$.

ICE analysis of outcome of INDELs. Genomic DNA was extracted using Nucleospin ${ }^{\circledR}$ Tissue (Macherey-Nagel, Duren, Germany) according to the manufacturer's protocol. The genomic region flanking $H B B$ exon 1, HBG2 and HBG1 promoter, and the $5^{\prime}$ breakpoint of the Corfu deletion were amplified by PCR (HotStarTaq Plus DNA polymerase, Qiagen ${ }^{\circledR}$, Venlo, Netherlands) with adequate primers (Supplementary Table 4). PCR products were purified with Nucleospin ${ }^{\circledR}$ Gel and PCR Clean-up (Macherey-Nagel) and sequenced (LIGHTRUN, GATC Biotech, Konstanz, Germany). The Inference of CRISPR Editing (ICE) software (https://ice.synthego.com) was used to determine the percentage of indels among polyclonal populations for all protocols allowing gRNA efficiencies by ICE
(Synthego). Non-transfected HEK293T and primary CD34 ${ }^{+}$cells are provided as control chromatogram. The limit of quantification (loq) was set at $2 \%$.

Cytogenetic examination of chromosomes 11 and 2. To validate the SNP loss screening method and visualize chromosomal instability, FISH was performed on interphase nuclei with probes targeting the following regions on Chr11: subcentromeric region (XCE 11 probe, labeled in orange) (MetaSystems Probes, Altlussheim, Germany), and sub-telomeric regions ( $\mathrm{p}$-arm sub-telomere probes labeled in green) (Cytocell Ltd, Cambridge, UK). In parallel, Chr2 was analyzed by FISH as control. FISH was performed on interphase nuclei with probes targeting the following regions on Chr2: sub-centromeric region (XCE 2 probe, labeled in green) (MetaSystems Probes, Altlussheim, Germany), and sub-telomeric regions (q-arm sub-telomere probes labeled in red (Texas red)) (Cytocell Ltd, Cambridge, UK).

Preparations were pre-treated as indicated below. Briefly, cells were first lysed by hypotonic shock using a $2.8 \mathrm{G} / \mathrm{L} \mathrm{KCl}$ solution, then fixed using a $3 / 4$ methanol $1 / 4$ acetic acid solution and deposited onto Super Frost microscopy slides (Thermo Scientific ${ }^{\mathrm{TN}}$ ). The slides were successively immersed in a $2 \mathrm{x}$ salinesodium citrate buffer for $10 \mathrm{~min}$ at $37^{\circ} \mathrm{C}$, in a $0.01 \%$ pepsin solution for $10 \mathrm{~min}$ at $37^{\circ} \mathrm{C}$, in a $1 \mathrm{x}$ phosphate-buffered saline (PBS) solution for $5 \mathrm{~min}$, in a $3.7 \%$ formaldehyde solution for $5 \mathrm{~min}$, and finally in a $1 \mathrm{x}$ PBS solution for $5 \mathrm{~min}$. Then they were dehydrated by immersing them successively for $1 \mathrm{~min}$ in four coplins of alcohol $70^{\circ}, 80^{\circ}, 90^{\circ}$, and $100^{\circ}$. FISH probes and DNA were then co-denaturated according to the manufacturers' protocols, and hybridization was performed overnight at $37^{\circ} \mathrm{C}$. The slides were then immersed successively in wash solutions and the nucleic acids were counterstained by 4,6-diamidino-2phenylindole (DAPI). The slides were then placed under an Axio Imager-2 microscope with an epi-fluorescence source (Carl Zeiss AG, Oberkochen, Germany). The microscope was linked to the Metafer 4 software for automated image acquisition and processing (MetaSystems $\mathrm{GmbH}$, Altlussheim, Germany). Magnification factor X630.

Array-CGH and SNP array. Array-CGH was performed on $8 \times 60 \mathrm{k}$ oligonucleotide microarrays, SNP+ CGH Array was performed on Genetisure Cyto $180 \mathrm{~K} \mathrm{CGH/}$ SNP arrays (Agilent Technologies, CA). DNA was labeled (cyanine 3 or cyanine 5) using the Genomic DNA ULS Labeling Kit from Agilent Technologies and hybridized onto the microarrays according to the manufacturer's instructions (Agilent).For SNP+ CGH arrays, tested DNAs were hybridized against male control DNA obtained from Agilent. Scanning of the microarrays was performed using a G2565CA scanner (Agilent). Data analysis was carried out with Agilent Technologies software, namely Feature Extraction for Cytogenomics Algorithm V5.0.1.16 for the fluorescence ratio calculation and Agilent CytoGenomics 4.0 and 5.0 to visualize chromosomal imbalances and loss of heterozygosity ( $\mathrm{LOH}$ ). Deletions and duplications in the heterozygous state were characterized by values of the $\log 2$ ratio of fluorescence intensities (cyanine 5/cyanine 3) below -0.5 and above +0.3 , respectively, with the statistical algorithm ADM 2 used at a threshold of 5. LOH was evaluated with the statistical algorithm ADM2 used at a threshold of 6 (Default Analysis Method v2).

Off-target analysis. The Top-10-predicted off-target sites identified by CRISPOR software were screened to evaluate a putative off-target in Chr11 (Supplementary Table 2).

Methylation analyses. Bisulfite conversion of $250 \mathrm{ng}$ of genomic DNA was performed using the EpiTect Bisulfite Kit according to the manufacturer's instructions (QIAGEN, Hilden, Germany). Methylation was then analyzed using real-time quantitative methylation-specific PCR (qMSP) ${ }^{39}$. Real-time quantitative PCR 
amplification conditions were $95^{\circ} \mathrm{C}$ for $3 \mathrm{~min}$, followed by 45 cycles at $95^{\circ} \mathrm{C}$ for $30 \mathrm{~s}, 62{ }^{\circ} \mathrm{C}$ for $30 \mathrm{~s}$, and $66^{\circ} \mathrm{C}$ for $30 \mathrm{~s}$, using a SyberGreen Master Mix (ROCHE Molecular Diagnostics), and a LC480 qPCR machine (ROCHE Molecular Diagnostics). The primers used for qMSP and BGS analyses were designed using the MethPrimer website [https://www.urogene.org/cgi-bin/methprimer/methprimer. cgi] and the OLIGO 4.0 software (Molecular Biology Insights, Inc., Colorado Springs, CO, USA). Primer sequences for the $H 19$ proximal promoter were: Methylated alleles: Forward: CGTTTGTTAGTAGAGTGCGTTCG, Reverse: AAC CGAACTTATACTCGTCACCG; Unmethylated alleles: Forward: TATTGTTTGT TAGTAGAGTGTGTTTG, Reverse: ATTAACCAAACTTATACTCATCACCA. Primer sequences for the H19 DMR (differentially methylated region) were: Methylated alleles: Forward: CGGTTTTATCGTTTGGATGGTAC, Reverse: CGA CGCGTAACTTAAATAACCCG; Unmethylated alleles: Forward: TTTTGGTTTT ATTGTTTGGATGGTAT, Reverse: CTACAACACATAACTTAAATAACCCA. Primer sequences for the IGF2 DMR (differentially methylated region) were: Methylated alleles: Forward: ATTTTTTTAGGAAGTATAGTTACGTC, Reverse: ATAAAAAATACACACGAATAACCCG; Unmethylated alleles: Forward: TTT ATTTTTTTAGGAAGTATAGTTATGTT, Reverse: AAATAAAAAATACACACA AATAACCCA. Primer sequences for the KCNQ1OT1 (KCNQ1 opposite strand/ antisense transcript 1) DMR (differentially methylated region) were: Methylated alleles: Forward: GTTTATTATTTCGGGGTGATCGC, Reverse: CTAATCTCGAA CGTAACCTAAACG; Unmethylated alleles: Forward: GTGTTTATTATTTTGGG GTGATTGT, Reverse: AACTAATCTCAAACATAACCTAAACA. Quantitative methylation-specific PCR (qMSP) analyses were quantified using the comparative MNE (mean normalized expression) method ${ }^{39}$, here adapted to copy numbers of unmethylated and methylated DNA alleles. Data are displayed as percentage of unmethylated and methylated alleles. PCR specificity was verified by melting curves, gel electrophoresis and Sanger sequencing analyses.

DNA from wild-type CD34 ${ }^{+}$cells and CRISPR-Cas9 clones 34.8 and 34.15 with iCN-LOH were analyzed by quantitative real-time methylation-specific PCR (qMSP). Wild-type peripheral blood leukocytes (PBL) from healthy donors, in vitro methylated DNA and No DNA were used as negative and positive controls. Percentages of unmethylated and methylated alleles (mean \pm SEM from independent measurements) are shown.

Gene expression analysis. Gene expression levels were determined by reverse transcription/real-time quantitative PCR (qRT-PCR), using the comparative MNE (Mean Normalized Expression) method ${ }^{40}$. The primers used for qRTPCR analyses were $H 19$-forward: CGTCCCTTCTGAATTTAATTTG, $H 19$-reverse: ACACTCGTACTGAGACTC, IGF2- forward: GTCGCAGCCGTGGCATCGTT, IGF2-reverse: AGGTGTCATATTGGAAGAACTTG. To normalize the qRT-PCR data, we used the primers for the following housekeeping genes: TATA box binding protein (TBP): TBP-forward: AGTGAAGAACAGTCCAGACTG, TBP-reverse: CCAGGAAATAACTCTGGCTCAT; Glyceraldehyde-3-phosphate dehydrogenase (GAPDH): GAPDH-forward: CTGCACCACCAACTGCTTAG, GAPDH-reverse: AGGTCCACCACTGACACGTT; and eukaryotic translation elongation factor 1 alpha 1 (EEF1A1/EF1 $\alpha$ ): EF1 $\alpha$-forward: CTGGAGCCAAGTGCTAACATG, EF1 $\alpha$ reverse: CCGGGTTTGAGAACACCAGT. Similar results were obtained when normalizing with these three housekeeping genes. Melting curves, gel electrophoresis and sequencing analyses showed that these primers amplified only the specific fragments.

Statistical analysis. Statistical significance was inferred when necessary. Graph Pad Prism 6 software was used for statistical analysis. Results are presented as mean \pm SEM (standard error of the mean). Two-sided chi-squared tests were used to compare percentages. All comparisons are shown with black bars. Null hypothesis was rejected when $p$-value $<0.05$. ns, non-significant.

Reporting summary. Further information on research design is available in the Nature Research Reporting Summary linked to this article.

\section{Data availability}

The source data generated in this study have been deposited in the ZENODO database https://doi.org/10.5281/zenodo.5130579.

Received: 24 November 2020; Accepted: 29 July 2021; Published online: 13 August 2021

\section{References}

1. Rouet, P., Smih, F. \& Jasin, M. Expression of a site-specific endonuclease stimulates homologous recombination in mammalian cells. Proc. Natl Acad. Sci. USA 91, 6064-6068 (1994)

2. Jinek, M. et al. A programmable dual-RNA-guided DNA endonuclease in adaptive bacterial immunity. Science 337, 816-821 (2012).
3. Cong, L. et al. Multiplex genome engineering using CRISPR/Cas systems. Science 338, 819-823 (2013)

4. Brunet, E. et al. Chromosomal translocations induced at specified loci in human stem cells. Proc. Natl Acad. Sci. USA 106, 10620-10625 (2009).

5. Thomas, M., Burgio, G., Adams, D. J. \& Iyer, V. Collateral damage and CRISPR genome editing. PLoS Genet. 15, e1007994 (2019).

6. Owens, D. D. G. et al. Microhomologies are prevalent at Cas9-induced larger deletions. Nucleic Acids Res. 47, 7402-7417 (2019)

7. Park, S. H. et al. Highly efficient editing of the $\beta$-globin gene in patient-derived hematopoietic stem and progenitor cells to treat sickle cell disease. Nucleic Acids Res. 47, 7955-7972 (2019).

8. Shin, H. Y. et al. CRISPR/Cas9 targeting events cause complex deletions and insertions at 17 sites in the mouse genome. Nat. Commun. 8, 15464 (2017).

9. Adikusuma, F. et al. Large deletions induced by Cas9 cleavage. Nature $\mathbf{5 6 0}$, E8-E9 (2018).

10. Kosicki, M., Tomberg, K. \& Bradley, A. Repair of double-strand breaks induced by CRISPR-Cas9 leads to large deletions and complex rearrangements. Nat. Biotechnol. 36, 765-771 (2018).

11. Alanis-Lobato, G. et al. Frequent loss of heterozygosity in CRISPR-Cas9edited early human embryos. Proc. Natl Acad. Sci. USA 118, e2004832117 (2021).

12. Cullot, G. et al. CRISPR-Cas9 genome editing induces megabase-scale chromosomal truncations. Nat. Commun. 10, 1136 (2019).

13. Przewrocka, J., Rowan, A., Rosenthal, R., Kanu, N. \& Swanton, C. Unintended on-target chromosomal instability following CRISPR/Cas9 single gene targeting C. Ann. Oncol. 31, 1270-1273 (2020).

14. Weisheit, I. et al. Detection of deleterious on-target effects after HDRmediated CRISPR editing. Cell Rep. 26, 107689 (2020).

15. Zuccaro, M. V. et al. Allele-specific chromosome removal after Cas9 cleavage in human embryos. Cell 29, 31389-1 (2020).

16. Vitale, I., Manic, G., De Maria, R., Kroemer, G. \& Galluzzi, L. DNA damage in stem cells. Mol. Cell. 66, 306-319 (2017).

17. Ihry, R. et al. p53 inhibits CRISPR-Cas9 engineering in human pluripotent stem cells. Nat. Med. 24, 939-946 (2018).

18. Haapaniemi, E., Botla, S., Persson, J., Schmierer, B. \& Taipale, J. CRISPR-Cas 9 genome editing induces a p53-mediated DNA damage response. Nat. Med. 24, 927-930 (2018)

19. Traxler, E. A. et al. A genome-editing strategy to treat $\beta$-hemoglobinopathies that recapitulates a mutation associated with a benign genetic condition. Nat. Med. 22, 987-990 (2016).

20. Antoniani, C. et al. Induction of fetal hemoglobin synthesis by CRISPR/Cas9mediated editing of the human $\beta$-globin locus. Blood 131, 1960-1973 (2018).

21. Hastings, R. J. et al. Guidelines for cytogenetic investigations in tumours. Tumour Best Practice meeting; Eurogentest. Eur. J. Hum. Genet. 24, 6-131 (2016).

22. Wilkin, F. et al. H19 sense and antisense transgenes modify insulin-like growth factor-II mRNA levels. Eur. J. Biochem. 267, 4020-4027 (2000).

23. Monk, D. et al. Genomic imprinting disorders: lessons on how genome, epigenome and environment interact. Nat. Rev. Genet. 20, 235-248 (2019).

24. Tuna, M., Knuutila, S. \& Mills, G. B. Uniparental disomy in cancer. Trends Mol. Med. 15, 120-128 (2009)

25. O'Keefe, C. et al. Copy neutral loss of heterozygosity: a novel chromosomal lesion in myeloid malignancies. Blood 115, 2731-2739 (2010).

26. Davis, L. et al. POLQ suppresses interhomolog recombination and loss of heterozygosity at targeted DNA breaks. Proc. Natl Acad. Sci. USA 15, 22900-22909 (2020).

27. Ma, H. et al. Correction of a pathogenic gene mutation in human embryos. Nature 24, 413-441 (2017).

28. Henry, I. et al. Uniparental paternal disomy in a genetic cancer-predisposing syndrome. Nature 20, 35 (1991).

29. Wu, Y. et al. Highly efficient therapeutic gene editing of human hematopoietic stem cells. Nat. Med. 25, 776-783 (2019).

30. Enache, O. M. et al. Cas 9 activates the p53 pathway and selects for p53inactivating mutation. Nat. Genet. 52, 748-749 (2020).

31. Kramara, J., Osia, B. \& Malkova, A. Break-induced replication: the where, the why, and the how. Trends Genet. 34, 518-531 (2018).

32. Pham, N. et al. Mechanisms restraining break-induced replication at twoended DNA double-strand breaks. EMBO J. 40, e104847 (2021).

33. Javidi-Parsijani, P. et al. CRISPR/Cas9 increases mitotic gene conversion in human cells. Gene Ther. 27, 281-296 (2020).

34. Leibowitz, M. L. et al. Chromothripsis as an on-target consequence of CRISPR-Cas9 genome editing. Nat. Genet. 53, 895-905 (2021).

35. Rees, H. A. \& Liu, D. R. Base editing: precision chemistry on the genome and transcriptome of living cells. Nat. Rev. Genet. 19, 770-788 (2018).

36. Anzalone, A. V., Koblan, L. W. \& Liu, D. R. Genome editing with CRISPR-Cas nucleases, base editors, transposases and prime editors. Nat. Biotechnol. 38, 824-844 (2020). 
37. Prat, F. et al. Mutation-Specific Guide RNA for Compound Heterozygous Porphyria On-target Scarless Correction by CRISPR/Cas9 in Stem Cells. Stem Cell Rep. 15, 677-693 (2020).

38. Charaf, L. et al. Effect of tyrosine kinase inhibitors on stemness in normal and chronic myeloid leukemia cells. Leukemia 31, 65-74 (2017).

39. Lo, Y. M. et al. Quantitative and temporal correlation between circulating cellfree Epstein-Barr virus DNA and tumor recurrence in nasopharyngeal carcinoma. Cancer Res. 59, 3899-3903 (1999).

40. Simon, P. Q-Gene: processing quantitative real-time RT-PCR data. Bioinformatics 19, 1439-1440 (2003).

\section{Acknowledgements}

V. Pitard, from the cytometry platform; Ligue Régionale Contre le Cancer and Association Française contre les Myopathies Grant \#22523 and ANR 2021 CRISPR-genotox for funding; V. Prouzet-Mauléon, INSERM U1218, Bordeaux, France for helpful discussion; N. Faur, Tumor Biology and Tumor Bank Laboratory, University Hospital Bordeaux, France, for help with bisulfite conversion of DNA and advice on real-time PCR equipment for methylation analyses. We thank Maison de Santé Protestante de Bordeaux-Bagatelle Hospital for providing umbilical cord-blood samples.

\section{Author contributions}

J. Boutin, J.R., F.M.G., and A.B. designed study, analyzed data, and wrote manuscript. A.B., F.M.G. supervised study and grants. D.C. hypothesized implication of imprinting, designed DNA-methylation analyses, qRT-PCR of $\mathrm{H} 19$ and IGF2 performed and interpreted experiments, and wrote corresponding parts of manuscript. J.P.M. and S.A. performed DNA methylation analyses and qRT-PCR in their laboratory and provided helpful advice. I.L.G. prepared CD34+ ${ }^{+}$cells. J.M.B., E.R., G.C., and S.D. contributed to study design. J.T., J. Boutin designed and performed FISH experiments. J.R., J. Boutin performed editing experiments and cell culture. P.P., C.R., and J. Bouron conducted array-CGH and CGH + SNP microarray analyses. All authors edited and approved final manuscript.

\section{Competing interests}

The author declares no competing interests.

\section{Additional information}

Supplementary information The online version contains supplementary material available at https://doi.org/10.1038/s41467-021-25190-6.

Correspondence and requests for materials should be addressed to F.M-G.

Peer review information Nature Communications thanks the anonymous reviewer(s) for their contribution to the peer review of this work.

Reprints and permission information is available at http://www.nature.com/reprints

Publisher's note Springer Nature remains neutral with regard to jurisdictional claims in published maps and institutional affiliations.

(c) (i) Open Access This article is licensed under a Creative Commons Attribution 4.0 International License, which permits use, sharing, adaptation, distribution and reproduction in any medium or format, as long as you give appropriate credit to the original author(s) and the source, provide a link to the Creative Commons license, and indicate if changes were made. The images or other third party material in this article are included in the article's Creative Commons license, unless indicated otherwise in a credit line to the material. If material is not included in the article's Creative Commons license and your intended use is not permitted by statutory regulation or exceeds the permitted use, you will need to obtain permission directly from the copyright holder. To view a copy of this license, visit http://creativecommons.org/ licenses/by/4.0/.

(C) The Author(s) 2021 